\title{
Intramyocardial Injected Human Umbilical Cord- Derived Mesenchymal Stem Cells (Hucmscs ) Contribute to the Recovery of Cardiac Function and the Migration of CD4+ T Cells into the Infarcted Heart via CCL5/CCR5 Signaling
}

Jing Liu

Shanghai East Hospital

Xiaoting Liang

Shanghai East Hospital

Mimi Li

Shanghai East Hospital

Fang Lin

Shanghai East Hospital

Xiaoxue Ma

Shanghai East Hospital

Yuanfeng Xin

Shanghai East Hospital

Qingshu Meng

Shanghai East Hospital

Rulin Zhuang

Shanghai East Hospital

Qingliu Zhang

Shanghai East Hospital

Wei Han

Shanghai East Hospital

Zhiying He

Shanghai East Hospital

Xiaohui Zhou ( $\square$ zxh100@tongji.edu.cn )

Shanghai East Hospital https://orcid.org/0000-0002-9354-3789

Zhongmin Liu

Shanghai East Hospital 
Keywords: Human umbilical cord-derived mesenchymal stem cells, intramyocardial injection, myocardial infarction, CD4+ T cells, CCL5/CCR5

Posted Date: December 15th, 2021

DOI: https://doi.org/10.21203/rs.3.rs-1133603/v1

License: (c) (1) This work is licensed under a Creative Commons Attribution 4.0 International License. Read Full License 


\section{Abstract}

Background: Human umbilical cord-derived mesenchymal stem cells (HucMSCs) have been recognized as a promising cell for treating myocardial infarction (MI). Inflammatory response post $\mathrm{Ml}$ is critical in determining the cardiac function and subsequent adverse left ventricular remodeling. However, the local inflammatory effect of HucMSCs after intramyocardial injection in murine remains unclear.

Methods: HucMSCs were cultured and transplanted into the mice after MI surgery. Cardiac function, angiogenesis, fibrosis and hypertrophy, and immune cells infiltration were evaluated between MI-N.S and MI-HucMSC groups. We detected the expression of inflammatory cytokines and their effects on $\mathrm{CD} 4^{+} \mathrm{T}$ cells migration.

Results: HucMSCs treatment can significantly improve the cardiac function and some cells can survive at least 28 days after MI. Intramyocardial administration of HucMSCs also improved angiogenesis and alleviated cardiac fibrosis and hypertrophy. Moreover, we found the much higher numbers of $\mathrm{CD} 4^{+} \mathrm{T}$ cells and $\mathrm{CD} 4^{+} \mathrm{FoxP}^{+}$regulatory T cells in the heart with HucMSC than that with N.S treatment on day 7 post MI. In addition, the protein level of C-C Motif Chemokine Ligand 5 (CCL5) greatly increased in the HucMSCs treated heart compared to the control. In vitro, HucMSCs inhibited CD $4^{+}$T cells migration and addition of CCL5 antibody or C-C Motif Chemokine receptor 5 (CCR5) antagonist significantly reversed this effect.

Conclusion: These findings indicated that HucMSCs contributed to cardiac functional recovery and attenuated cardiac remodeling post MI. Intramyocardial injection of HucMSCs upregulated the $\mathrm{CD} 4^{+} \mathrm{FoxP}^{+}$regulatory $\mathrm{T}$ cells and contributed to the migration of $\mathrm{CD} 4^{+} \mathrm{T}$ cells into the injured heart via CCL5/CCR5 pathway.

\section{Highlights}

1. HucMSCs with intramyocardial injection can survive in murine myocardium for at least 28 days and improve cardiac function post MI.

2. HucMSCs treatment contributed to the angiogenesis and attenuated cardiac fibrosis and hypertrophy in murine $\mathrm{Ml}$ models.

3. Intramyocardial administration of HucMSCs upregulated the $\mathrm{CD}^{+}{ }^{+} \mathrm{FoxP} 3^{+}$Tregs and contributed to the migration of $\mathrm{CD} 4^{+} \mathrm{T}$ cells into the injured heart via CCL5/CCR5 pathway.

\section{Introduction}

Cardiovascular diseases, with high prevalence, threaten human health and cause great economic burden. Myocardial infarction (MI) is a common cardiovascular disease, which refers to the severe and persistent ischemic necrosis of the myocardium after the coronary artery is interrupted [1]. Although current clinical treatments (such as percutaneous coronary intervention/PCI and coronary artery bypass graft/CABG) 
have some therapeutic effects, the disability and mortality of MI patients are still high due to extensive myocardial injury and poor prognosis [2]. Thus, it is essential to seek effective strategies to improve heart function and repair myocardium post ischemic injury.

Transplantation of mesenchymal stem cells (MSCs), representing one of the promising cell therapeutic strategies in Ml, has attracted great attention recently [3]. Various sources of MSCs including those derived from bone marrow, adipose tissue, perinatal tissues, and pluripotent stem cells have been examined in animal models as well as in preclinical and clinical trials. Umbilical cord as a kind of perinatal tissue, can be an attainable MSC source. Compared with adult tissue derived MSCs, human umbilical cord-derived MSCs (HucMSCs) has longer telomerase and activity, higher yield, shorter doubling time and relatively lower immunogenicity [4,5]. Recent studies confirmed the protective function of HucMSCs in acute MI animal models either by immediate intracoronary delivery [6], intravenous injection [7] or direct intramyocardial injection [5]. In two clinical trials [8], delayed intracoronary HucMSCs transfer was used in reperfused acute MI. These preliminary results indicated the safety and efficacy of HucMSCs in acute Ml.

Exaggerated or persistent inflammatory activation after MI leads to poor adaptive healing and subsequent left ventricular remodeling [9]. Accumulating evidences suggested that MSC therapy can regulate the balance between inflammation and repair after MI $[6,10]$. Intravenous delivery of human bone marrow-derived MSCs (BMSCs) demonstrated a systemic anti-inflammatory activity [10]. Recent study showed that intracoronary administration of HucMSCs regulate T cells mediated inflammation and reduce cardiac injury [6]. In the present study, we investigated how intramyocardial administration of HucMSCs regulate the immune response in the injured heart and contribute to cardiac healing in murine MI models.

\section{Materials And Methods}

\section{Ethic approval}

All experiments involving mice were approved by the Institutional Animal Care and Use Committee of Tongji University (Shanghai, China; approval no: TJLAC-019-131). The human umbilical cords were obtained from full-term births and implemented following the approval of Ethic Committee and the informed consent of donors in Shanghai East Hospital.

\section{Cell culture}

HucMSCs were isolated and cultured in good manufacturing practices (GMP) lab. In this experiment, the culture procedures of primary HucMSCs were as follows. The umbilical cord was placed in the $10 \mathrm{~cm}$ dish and cut into 2-3 cm tissue. Blood from the umbilical cord was washed and umbilical vein and artery were removed. The Wharton's jelly was separated from the umbilical cord and cut into pieces (approximately $1 \mathrm{~cm}$ ). Then, pieces of tissue were tiled and cultured in Minimum Essential Medium, Alpha 
(a-MEM, Corning, 10-022) supplemented with 5\% UltraGRO-Advanced (Helios, HPCFDCGL50), and were incubated at $37^{\circ} \mathrm{C}$ in a humidified atmosphere of $95 \%$ air and $5 \% \mathrm{CO}_{2}$ until HucMSCs slowly crawled out of pieces. After that, the medium was changed every 2-3 day, and HucMSCs underwent passages using Trypin-Express (Gibco, 12604021) solution. HucMSCs were used at passage 4-5 (P4-5) for all experiments.

\section{Tumorigenicity evaluation of HucMSCs in vivo}

To confirm the tumorigenicity of HucMSCs in vivo, nude mice were randomly (using computer-generated random numbers) divided into two groups named HucMSC and A549 groups, and injected subcutaneously into the left arm with $1 * 10^{6}$ HucMSCs or A549 cells, respectively. The survival situation of each group was evaluated for 120 days.

\section{Multiple enzymes detection}

After HucMSCs intramyocardial injection, blood (about $300 \mu \mathrm{L}$ ) from different groups was collected on day 7 and 28, and the serum was isolated by centrifugation. Aspartate transaminase (AST), Alanine aminotransferase (ALT), Creatine (Cr), Lactate dehydrogenase (LDH), Creatine kinase (CK), Creatine kinase isoenzyme (CK-MB) levels were measured using Beckman AU680 (Beckman Coulter, Inc.) according to manufacturer's instructions.

\section{Histological analysis}

After euthanizing animals, mice tissues were fixed in 4\% paraformaldehyde (PFA) overnight, embedded in paraffin and sectioned at 3-4 $\mu \mathrm{m}$ intervals. The sections were stained with Hematoxylin and eosin (H\&E) and Masson's Trichrome. For Masson's Trichrome, fibrosis was quantified as the relative area of blue staining compared the left ventricle surface, as an average of six sections per heart at the level of the papillary muscle, using Image J software (National Institutes of Health).

\section{MI model establishment and HucMSCs injection}

The 8-10 weeks old male mice (C57BL/6J; 24-28 g) were purchased from SLAC Laboratory Animal Co., Ltd (Shanghai, China) and were housed in the specific pathogen free (SPF) environment. The mice were bred at $20-25^{\circ} \mathrm{C}$ at $12 \mathrm{~h}$ light/dark cycles, and given sterile water and food.

Briefly, animals were randomly (using computer-generated random numbers) divided into two groups ( $n=13$, per group): (1) MI-N.S group, intramyocardial injection of normal saline (N.S); (2) MI-HucMSC group, intramyocardial injection of HucMSCs. Before establishing MI models, HucMSCs were counted and resuspended in N.S at a final concentration of $3^{*} 10^{5}$ cells per mouse. Next, mice were anesthetized 
with sodium pentobarbital ( $60 \mathrm{mg} / \mathrm{kg}$ body weight, intraperitoneal injection), and then intubated and mechanically ventilated using a rodent respirator (Shanghai Alcott Biotechnology Co., Ltd, ALC-V8S). A left thoracotomy at the fourth-fifth intercostal space was performed, and the left coronary artery was ligated. Then, mice were immediately injected $30 \mu \mathrm{L}$ of $3^{*} 10^{5}$ HucMSCs or N.S by three points into the areas adjacent to the infarcted tissue $(10 \mu \mathrm{L}$ per point) with a 30 -gauge needle gas-tight syringe (Hamilton Company). The animal temperature was maintained during surgery by using a heating pad. Finally, the animals were euthanized after 7 or 28 days and tissues were collected for following experiments.

\section{Echocardiography}

Mice were anesthetized with $2 \%$ isoflurane inhalation and analyzed using a Vevo 2100 high-resolution imaging system with a 30-MHz linear transducer (FUJIFILM Visual-Sonics, Inc.) as previously described [11]. We acquired and analyzed the cardiac function of each group through long-axis scans using M-mode images including left ventricular ejection function (LVEF), left ventricular fractional shortening (LVFS), left ventricular end diastolic/systolic volume (LVEDV/LVESV) and left ventricular end diastolic/systolic diameter (LVEDD/LVESD) on day 7 and 28.

\section{Fluorescent labeling of HucMSCs}

HucMSCs at P3 were labeled with letivirus containing green fluorescence protein (GFP) at a multiplicity of infection of 10 as previously described [12]. Infection efficiency was confirmed by GFP fluorescent signal viewed under the microscope.

\section{Bioluminescence imaging in vivo}

HucMSCs were pre-stained with the near-infrared fluorescent-lipophilic tracer 1,1-dioctadecyl-3,3,3,3tetramethylindotricarbocyanine iodide (DiR, ThermoFisher, D12731) according to the manufacturer's instructions, and then $3^{*} 10^{5}$ DiR-labeled HucMSCs were injected in the heart of mice after MI operation. The fluorescence intensity of multiple organs (including heart, lung, liver, kidney and spleen) was analyzed using the IVIS imaging instrument (Xenogen, USA) with the relevant channel.

\section{Polymerase chain reaction (PCR)}

Genomic PCR for human Alu-sx repeat sequences in heart tissue was performed as previously reported [13]. The primer of human Alu-sx is: F:5'-GGCGCGGTGGCTCACG-3', R:5'-

TTTTTTGAGACGGAGTCTCGCTC-3'. The amplified products were determined by electrophoresis in $1.5 \%$ agarose gel supplemented with ethidium bromide. 


\section{Immunofluorescence staining}

Firstly, frozen sections were fixed with $4 \%$ PFA and blocked with $1 \%$ Bovine Serum Albumin (BSA) at $4^{\circ} \mathrm{C}$ for $30 \mathrm{~min}$. Then, the sections were incubated with wheat germ agglutinin (WGA, Thermo Fisher, W11261), anti-CD31 (Cell Signaling, 3528S), anti-CD3 (Abcam, ab231775), anti-CD4 (Abcam, ab183685), anti-FoxP3 (Abcam, ab215206), anti-Ki67 (Abcam, ab16667) and human mitochondrion antibodies (Abcam, ab92824), at $4^{\circ} \mathrm{C}$ overnight to visualize cardiac cells, immune cells and HucMSCs in heart tissue, respectively, while 2-(4-Amidinophenyl)-6-indolecarbamidine dihydrochloride (DAPI) was used to mark cell nuclei. After incubated with above antibodies and washed with phosphate buffered saline (PBS), different fields of each slide were randomly selected and photograph under an inverted fluorescent microscope (Leica DM6000B, Germany).

\section{Real-time quantitative PCR (RT-qPCR)}

According to the manufacturer's instruction, the total RNA was extracted with TRIzol regent (Beyotime, R0016), and then the concentration of total RNA was quantified with NanoDrop2000 (Thermo Fisher Scientific, Inc.). cDNA was obtained by reverse transcription PCR using the PrimerScript RT reagent kit (Takara, RR0036A) at $37^{\circ} \mathrm{C}$ for $15 \mathrm{~min}$, and $85^{\circ} \mathrm{C}$ for $5 \mathrm{sec}$. The mRNA levels were detected by RT-qPCR with a SYBR Green Master Mix kit (Thermo Fisher, 4385617) using an ABI QuantStudio 6 Flex System. The mRNA levels were normalized to the endogenous control GAPDH. The relative gene quantification was calculated using the $2^{-\triangle \Delta C T}$ method. Gene primers were listed in Table S1.

\section{Preparation of single cell and Flow cytometry analysis}

Briefly, mice were anesthetized with $1 \%$ sodium pentobarbital $(60 \mathrm{mg} / \mathrm{kg}$, intraperitoneal injection), and the heart was exposed and perfused with pre-cold PBS on day 7 after MI. Then, the heart was cut into $1 \mathrm{~mm}$ and put into the $C$-tube with collagenase II solution $(1.5 \mathrm{mg} / \mathrm{mL}$, added $500 x$ DNAse). During broken twice using gentleMACS ${ }^{\mathrm{TM}}$ Tissue Dissociators (Miltenyi Biotec, USA), the heart tissue was centrifuged at $37^{\circ} \mathrm{C}$ for $30 \mathrm{~min}$. After removing debris, the supernatant was filtered (70 $\mu \mathrm{m}$ strainer) to obtain single cell suspension. Cells were stained with the following labeled antibodies: anti-mouse CD45 (Biolegend, Clone 13/2.3), CD19 (Biolegend, Clone 1D3/CD19), CD3 (Biolegend, Clone 17A2), CD4 (Biolegend, Clone GK1.5), F4/80 (Biolegend, Clone BM8), Ly-6G (Biolegend, Clone 1A8). Data were acquired on a FACS Beckman flow cytometer (BD Biosciences, USA), and analysis was performed with Flow Jo software (BD Biosciences, USA).

\section{Cytokine profiling detection}

For cytokine protein expression, the tissue samples of infarct and border area in heart of MI murine were collected and weighed. Then we added $500 \mu \mathrm{L}$ ProcartaPlex Cell Lysis Buffer (EPX-99999-000) per 100 
$\mathrm{mg}$ tissue. After tissue homogenization and centrifugation, the protein of sample was quantified by Bicinchoninic Acid (BCA) Protein Assay Kit (Bio-Rad, Hercules, CA, USA) and then detected for cytokine profiling (Thermo Fisher, EPX110-20820-901, EPX01A-20614-901, EPX01A-26005-901 and EPX01A26009-901).

\section{The purification and culture of murine $\mathrm{CD}^{+} \mathrm{T}$ cells from spleen}

First, RPMI 1640 complete medium (FBS 5 mL; PS $500 \mu \mathrm{L}$; HEPES buffer solution $500 \mu \mathrm{L}$; Nonessential Amino Acids $500 \mu \mathrm{L}$; Sodium Pyruvate solution $500 \mu \mathrm{L}$ ) and magnetic bead buffer (PBS $48.5 \mathrm{~mL}$; FBS 1 $\mathrm{mL} ; 1 \mathrm{mM}$ EDTA $500 \mu \mathrm{L}$ ) were prepared. Next, the spleen was clipped and ground after the mice were anesthetized. Then, primary $\mathrm{CD} 4^{+} \mathrm{T}$ cells in the spleen were isolated by immunomagnetic bead (Thermo Fisher, $11415 \mathrm{D})$. The density of $\mathrm{CD} 4^{+} \mathrm{T}$ cells was adjusted by cell count $\left(1 * 10^{6} \mathrm{cells} /\right.$ per well) and planted to 24 -well plate. $\mathrm{CD} 4^{+} \mathrm{T}$ cells were cultured with RPMI1640 complete medium and stimulated with anti-CD3/CD28 magnetic beads (cells:magnetic beads=5:1, Gibco 00702195).

\section{Enzyme-linked immunosorbent assay (Elisa)}

The concentrations of vascular endothelial growth factor (VEGF)- $a$ in the tissue supernatants were measured using Quantikine Elisa kits for VEGF-a (Multi Sciences, EK283/2), according to the manufacturer's instructions. All results were normalized to the total protein content.

\section{Cell migration assay}

Cardiac tissues containing infarct and border zone in MI-N.S and MI-HucMSC groups were collected and lysed and the protein quantified by BCA assay. The concentration of C-C Motif Chemokine Ligand 5 (CCL5) was quantified by Elisa assay (absin, abs520014) according to the manufacturer's instructions. Polycarbonate membrane transwell inserts ( 24 well, pore size $5.0 \mu \mathrm{m}$ ) were used for $\mathrm{CD} 4^{+} \mathrm{T}$ cell migration assay. At the lower chamber, a total volume of $500 \mu \mathrm{L}$ RPMI1640 containing $20 \mu \mathrm{g}$ tissue protein were added. At the upper chamber, a total number $5^{\star} 10^{5}$ purified $\mathrm{CD} 4^{+} \mathrm{T}$ cells were added. Sufficient dose CCL5 antibody (R\&D system, MAB478) or CCL2 antibody (R\&D system, AF-479-NA) was added to the lower chamber for neutralization with information of CCL5/CCL2 concentration determined by Elisa assay (For example, add $0.5 \mu \mathrm{g} / \mathrm{mL}$ of CCL5 antibody per $0.025 \mu \mathrm{g} / \mathrm{mL}$ of CCL5). Sufficient dose (10 ng/mL) C-C Motif Chemokine receptor 5 (CCR5) antagonist (MCE, HY-100261) or CCR2 antagonist (MCE, HY-108323) was added to the upper chamber with purified CD4 ${ }^{+} \mathrm{T}$ cells in indicated groups. After co-culturing for $16 \mathrm{~h}$, the $\mathrm{CD} 4^{+} \mathrm{T}$ cells that had migrated to the lower chamber were collected and counted by handheld automated cell counter (millipore). 


\section{Statistical analysis}

The study was performed using GraphPad analysis software (version 8, USA), and data were expressed as mean \pm Standard Error of Mean (SEM). Statistical significance of differences between two groups were compared by an unpaired two-tailed Student's T-test, while multiple comparsions were applied the log-rank test. The survival curve was estimated by the Kaplan-Meier Test. $P<0.05$ was defined as statistically significant.

\section{Results}

\section{The phenotype and safety evaluation of HucMSCs}

Firstly, flow cytometry analysis demonstrated that HucMSCs express the typical surface markers of CD73, CD105 and CD90, while CD11b, CD19, CD34, CD45 and HLA-DR are absent in these cells (Sup Fig 1A). Sup Fig 1B showed the tri-lineage including osteogenic, adipogenic and chondrogenic differentiation potential of HucMSCs.

Then, nude mice were applied to assess the safety of HucMSCs in vivo. After $1 * 10^{6}$ A549 cells injection, a subcutaneous tumor was occurred in the left arm of nude mouse on day 60 (Fig 1A). But no tumor formation was observed and no mice died in the HucMSC group for consecutive 120 days compared with A549 group (Fig 1B). H\&E staining results showed that intramyocardial injection of HucMSCs had no obvious impact on the pathology of multiple organs, including the lung, liver, kidney and spleen on day 28 (Fig 1C). In addition, intramyocardial treatment with HucMSCs did not affect the levels of ALT, AST, Cr in serum on day 7 and 28 nor affect LDH, CK and CK-MB on day 7 in murine MI models (Fig 1D).

\section{Intramyocardial administration of HucMSCs improved cardiac function post MI}

To determine whether HucMSCs exerted a protective role in murine MI models, $3 * 10^{5}$ HucMSCs were injected in the peri-infarct area. The flow chart of the experiment was shown in Fig 2A, and cardiac function was tested by M-mode echocardiography on day 7 and 28 after HucMSCs intramyocardial treatment. As shown by Fig $2 \mathrm{~B}$ to $2 \mathrm{E}$, administration of HucMSCs significantly improved LVEF and LVFS on both day 7 and 28, and further reduced LVEDV/LVESV and LVEDD/LVESD on day 28 compared to the N.S treated MI mice.

\section{Retention of HucMSCs in vivo after intramyocardial injection}


Next, we confirmed the retention of HucMSCs in vivo after MI. DiR-labeled HucMSCs were administrated into the myocardium, and high intensity of HucMSCs signaling were detected in heart, lung, liver and spleen in MI-HucMSC group on day 7, compared with MI-N.S group (Fig 3A). Fig 3B showed that human Alu-sx repeat sequences were found at the infarcted and border areas of heart in MI-HucMSC group on day 7 by genomic PCR, compared with MI-N.S group. Additionally, fluorescence results showed that green fluorescent signals were present in the border zone of the heart on day 7 when GFP-labeled HucMSCs were injected into the myocardium (Fig $3 \mathrm{C}$ ). And the immunofluorescence staining of human mitochondrion further verified the presence of HucMSCs in the myocardium at 28 days post MI (Fig 3D).

\section{HucMSCs treatment contributed to the myocardial angiogenesis but attenuated fibrosis and hypertrophy in murine MI models}

We further determined the role of HucMSCs on cardiac angiogenesis. As shown in Fig 4A and B, compared to the MI-N.S group, the expression of endothelial marker CD31 was significantly increased at the peri-infarct area in the MI-HucMSC group both on day 7 and 28. Moreover, HucMSCs treatment remarkably upregulated the protein level of vascular endothelial growth factor-a (VEGF- $a$ ) in the infarcted and border area of the heart on day 7 post MI (Fig 4C).

In addition, HucMSCs treatment significantly alleviated the average area of myocardium fibrosis, as compared with MI-N.S group on day 28 (Fig 4D and E). Meanwhile, compared to the MI-N.S heart, the expression of fibrosis markers including collagen III (COL III) and matrix metalloproteinase 2 (MMP2) significantly decreased in the MI heart treated with HucMSCs on day 28 (Fig 4F). Moreover, the immunofluorescence staining of WGA (Fig $4 \mathrm{G}$ and $\mathrm{H}$ ) and the mRNA expression of hypertrophy markers (included atrial natriuretic peptide/ANP, brain natriuretic peptide/BNP, myosin heavy chain 7/MYH7) (Fig 4I) together proved that HucMSCs administration attenuated cardiac hypertrophy on day 28 after MI.

\section{Increase of $\mathrm{CD}^{+}{ }^{+} \mathrm{T}$ cells and $\mathrm{CD} 4^{+} \mathrm{FoxP} 3^{+}$regulatory $\mathrm{T}$ cells in the infarcted heart after HucMSCs treatment}

The inflammatory response after acute $\mathrm{Ml}$ is critical in determining the infarcted size and subsequent adverse LV remodeling [14]. We next sought to verify whether administration of HucMSCs regulate the infiltration of immune cells into the heart on day 7. Firstly, large number of $\mathrm{CD}^{+} \mathrm{T}$ cells accumulated around the area of HucMSCs injection (Fig 5A). Furthermore, compared to the N.S group, both percentages of $\mathrm{CD}^{+}$and $\mathrm{CD} 4^{+} \mathrm{T}$ cells increased around the peri-infarcted area of heart in HucMSC treated group (Fig 5B, C). More importantly, the numbers of $\mathrm{CD}^{+}{ }^{+} \mathrm{FoxP} 3^{+}$regulatory $\mathrm{T}$ cells (Tregs) markedly upregulated in HucMSCs treated heart (Fig 5D, E). But, Figure 5F and G showed no significant difference in cells number of $\mathrm{CD} 4^{+} \mathrm{Ki} 67^{+} \mathrm{T}$ cells on day 7 between these two groups. 
Next, we digested the heart and analyzed the ratio and total numbers of immune cells in the heart (Fig $5 \mathrm{H})$. Compared with MI-N.S group, total numbers of single cells and $\mathrm{CD} 45^{+}$immune cells in the heart were increased in MI-HucMSC group through cell counts and calculating (total number multiply percentage) (Fig 5I). Then the large size of $\mathrm{CD} 45^{+}$immune cells were gated to analyze macrophages (CD $45^{+} \mathrm{F} 4 / 80^{+}$) and neutrophils $\left(C D 45^{+} \mathrm{Ly}-6 \mathrm{G}^{+}\right)$, while the small size of $\mathrm{CD} 45^{+}$immune cells were gated to analyze $\mathrm{B}$ cells $\left(\mathrm{CD} 45^{+} \mathrm{CD} 19^{+}\right)$and $\mathrm{T}$ cells $\left(\mathrm{CD} 45^{+} \mathrm{CD}^{+}\right)(\mathrm{Fig} 5 \mathrm{H})$. Flow cytometry results showed that the proportion of $\mathrm{CD} 45^{+} \mathrm{F} 4 / 80^{+}$macrophage and $\mathrm{CD} 45^{+} \mathrm{CD}^{+} \mathrm{T}$ cells in myocardial tissue were significantly enhanced in HucMSC treatment compared with the N.S control on day 7 after MI (Sup Fig 2). In particular, MI-HucMSC group significantly upregulated the percentage of $\mathrm{CD} 45^{+} \mathrm{CD} 3^{+} \mathrm{CD} 4^{+} \mathrm{T}$ cells but downregulated CD $45^{+} \mathrm{CD}^{+}{ }^{+} \mathrm{CD} 4^{-} \mathrm{T}$ cells as compared with those from MI-N.S group on day 7 post MI (Sup Fig 2). Similarly, the cell count of $\mathrm{CD} 45^{+} \mathrm{F} 4 / 80^{+}$macrophages, $\mathrm{CD} 45^{+} \mathrm{CD} 3^{+} \mathrm{T}$ cells and $\mathrm{CD} 45^{+} \mathrm{CD} 3^{+} \mathrm{CD} 4^{+} \mathrm{T}$ cells in cardiac tissue were higher in HucMSC than in N.S treated MI mice on day 7 by calculation (Fig $5 \mathrm{I}$ ).

\section{HucMSCs promoted CD4 ${ }^{+} \mathrm{T}$ cells migration into the injured heart via high levels of CCL5 post MI}

Then, we determined the cytokine profiling in the ischemic heart with different treatments. As shown in Sup Fig 3 and Fig $6 \mathrm{C}$, HucMSCs treatment significantly upregulated the protein levels of multiple cytokines and chemokines including IL-1 $\beta$, IL-4, IL-6, IL-12P70, Granulocyte macrophage-Colony Stimulating Factor (GM-CSF), tumor necrosis factor-a (TNF-a), CCL2 and CCL5 on day 7 in infarcted myocardium compared with the N.S control. There were no statistical differences of IL-2, IL-5, IL-10, IL13, IL-18 and Interferon-y (IFN-Y) expressions between MI-N.S and MI-HucMSC groups (Sup Fig 3). Among these molecules, CCL2 and CCL5 protein levels increased the most in the MI-HucMSC group (Fig 6A, B).

To further verify whether the infiltration of $C D 4^{+} \mathrm{T}$ cells into the HucMSCs treated heart was mediated via CCL2 and CCL5, primary CD4 ${ }^{+} \mathrm{T}$ cells from spleen were applied and co-cultured with heart homogenate from MI-N.S or MI-HucMSC group in vitro. Fig 6D showed that migrated cells number of $\mathrm{CD} 4^{+} \mathrm{T}$ cells significantly upregulated in MI-HuCMSC group, compared with control group. Addition of CCL5 antibody or CCR5 antagonist greatly suppressed the migration of $\mathrm{CD} 4^{+} \mathrm{T}$ cells induced by heart homogenate from MI-HucMSC group. Whereas both CCL2 antibody and CCR2 antagonist have no effect on $\mathrm{CD} 4^{+} \mathrm{T}$ cells migration (data not shown).

\section{Discussion}

MSC, as a promising cell candidate for transplantation, has been widely used in various clinical diseases especially ischemic heart diseases [15]. Recent clinical study demonstrated the safety of intramyocardial transplantation of HucMSCs in patients subjected to chronic ischemic cardiomyopathy by monitoring the incidence of adverse events within the 12 months follow-up period [16]. In the present study, we firstly evaluated the safety of HucMSCs in vivo. We did not find subcutaneous tumor formation nor murine 
death till 120 days after injection. There were no pathological changes in organs till day 28 after intramyocardial injection of HucMSCs. In addition, intramyocardial administration of HucMSCs did not affect the expression of multiple enzymes. These results primarily confirmed the safety of intramyocardial injection of HucMSCs to mice.

Results from preclinical studies indicated the role of HucMSCs in improving the cardiac function in acute rat MI models [17]. Further controlled, randomized clinical trial suggested the significant efficacy of intramyocardial delivery of HucMSCs in chronic ischemic cardiomyopathy [16]. Consistent with these findings, intramyocardial administration of HucMSCs after MI can significantly improve LVEF and LVFS on both day 7 and 28, and further reduced LVEDV/LVESV and LVEDD/LVESD on day 28 in murine MI models.

Despite the efficacy, the utilization of MSCs is still challenged because of their low engraftment and survival rate in recipient hearts. In porcine model of $\mathrm{Ml}, 6 \%$ of BMSCs by catheter-based transendocardial injection were tracked at 10 days [18]. Through positron emission tomography (PET) detection, only 1.3$2.6 \%$ BMSCs labeled with 18F-FDG were imaged after intracoronary injection at 50-75 minutes in MI patients [19]. Compared with intracoronary and interstitial retrograde coronary venous transplatation, intramyocardial injection had the most retention rate of BMSCs [20]. Our results further verified the presence of living HucMSCs after intramyocardial injection at least 28 days in murine infarcted myocardium.

Previous studies suggested the possible mechanisms by which HucMSCs improve cardiac function, including their transdifferentiation into cardiomyocyte-like cells by intramyocardial injection [21], the paracrine function contributing to angiogenesis, inflammation limitation, preservation of Cx43 gap junction by intravenous injection [7], and their regulation of fibrosis and apoptosis after intravenous administration [22]. Cardiomyocyte hypertrophy and interstitial fibrosis in the heart contribute to systolic and diastolic dysfunction, and ultimately to heart failure $[23,24]$. In the present study, we found that intramyocardial administration of HucMSCs significantly promote cardiac angiogenesis and attenuated cardiac hypertrophy and fibrosis compared to MI-N.S group on day 28 after MI. The improvement may partly explain the efficacy of HucMSCs on chronic cardiac dysfuntion on day 28.

Acute $\mathrm{Ml}$ is characterized by high levels of inflammatory responses, therefore, cardiac inflammation was reported to be an important therapeutic target [25]. Large amount of studies confirmed that inflammatory cells, especially $\mathrm{CD} 4^{+} \mathrm{T}$ cells, is closely associated with heart injury and wound healing $[25,26]$. Infiltration and activation of $\mathrm{CD} 4^{+} \mathrm{T}$ cells are essential for cardiac healing after $\mathrm{MI}$ in murine models [25]. Other studies showed the cellular interaction between MSCs and macrophages or T cells in cardiac injury and recovery $[6,27]$. Human umbilical cord perivascular MSCs treatment contribute to the switch from an inflammatory M1 macrophages to reparative M2 phenotype in acute MI [27]. The present study revealed that the levels of $C D 45^{+} C D 3^{+} T$ cells (especially $C D 45^{+} C D 3^{+} C D 4^{+} T$ cells) and $C D 45^{+} \mathrm{F} 4 / 80^{+}$ macrophages were increased in injury hearts after intramyocardial HucMSCs transplantation on day 7 after MI. We also found the adjacent locations of $\mathrm{CD}^{+}{ }^{+}$cells and HucMSCs in the border zone of the 
infarction. However, no significant difference of $\mathrm{CD} 4^{+} \mathrm{Ki} 67^{+} \mathrm{T}$ cells were found between MI-HucMSC and MI-N.S group. Therefore, administration of HucMSCs via intravenous may contribute to the infiltration of $T$ cells and macrophages into the injured heart.

Tregs, a subtype of $\mathrm{CD} 4^{+} \mathrm{T}$ cells known as an immune brake, can block excessive inflammatory responses and tissue destruction to maintain the homeostasis in vivo. Less recruitment of Tregs is associated with deteriorated LV dilation and increased expression of inflammatory mediators in the infarct zone in murine models [28]. And therapeutic activation or adoptive transfer of Foxp $3^{+} \mathrm{CD} 4^{+}$Tregs can improve cardiac healing after MI [9]. Intracoronary delivery of HucMSCs attenuate myocardial injury by promoting the generation of $\mathrm{CD} 4^{+} \mathrm{CD} 25^{+} \mathrm{Foxp}^{+}$Tregs [6]. Our results also revealed the marked increase of $\mathrm{CD}^{+}{ }^{+} \mathrm{Foxp}^{+}$Tregs in the heart post HucMSCs treatment. Therefore, intramyocardial injection HucMSCs may improve the cardiac function mainly through increasing the infiltration of $\mathrm{CD} 4^{+} \mathrm{T}$ cells as well as $\mathrm{CD}^{+}{ }^{+} \mathrm{Foxp}^{+}{ }^{+}$Tregs in the injured heart. Whether HucMSCs contribute to the differentiation of cardiac macrophages need further exploration.

Next, we explored which inflammatory cytokines signaling are involved in the recovery process of cardiac function and the migration of $\mathrm{CD} 4^{+} \mathrm{T}$ cells into the injured heart induced by HucMSCs. The cytokine profile indicated high levels of CCL2 and CCL5 in the HucMSCs treated heart. The migration results further confirmed the inhibition of CCL5 or CCR5 respectively reversed the up-regulation of the migrated cell number of $\mathrm{CD} 4^{+} \mathrm{T}$ cells induced by homogenate from heart in MI-HucMSC group in vitro. Consistently, previous study showed that MCP-1/CCL2 and RANTES/CCL5 regulated angiogenesis by stimulating endothelial cells branching after pathophysiologic process of ischemia [29]. Therefore, both CCL2 and CCL5 may contribute to the angiogenesis in infarct heart after HucMSCs injection.

RANTES/CCL5 is secreted by endothelial cells, smooth muscle cells, macrophages and platelet, and it can induce leucocyte recruitment and activate T cells [30]. Destruction of the CCR5 leads to adverse remodeling and cardiac deterioration, which is associated with decreased infiltration of Tregs [31]. Adipose derived-MSCs can upregulate the secretion of IL-8 and CCL5 to promote T lymphocyte recruitment when exposed to inflammatory environment [32]. Considering these reports, our results suggested that CCL5 signaling may partly mediated the effect of HucMSCs on the migration of $\mathrm{CD} 4^{+} \mathrm{T}$ cells and the increase of $\mathrm{CD} 4^{+} \mathrm{Foxp} 3^{+}$Tregs in the injured heart.

\section{Conclusion}

This study indicated that HucMSCs with intramyocardial injection can survive in murine myocardium post $\mathrm{Ml}$ at least 28 days. HucMSCs contributed to cardiac functional recovery and attenuated cardiac remodeling post MI. Intramyocardial injection of HucMSCs upregulated the $\mathrm{CD} 4^{+} \mathrm{FoxP} 3^{+}$Tregs and contributed to the migration of $\mathrm{CD} 4^{+} \mathrm{T}$ cells into the injured heart via CCL5/CCR5 pathway.

\section{Abbreviations}


MI (myocardiac infarct), HucMSCs (Human umbilical cord-derived mesenchymal stem cells), SEM (Standard Error of Mean), AST (aspartate transaminase), ALT (alanine aminotransferase), Cr (Creatine), LDH (Lactate dehydrogenase), CK (Creatine kinase), CK-MB (Creatine kinase isoenzyme), LVEF (left ventricular ejection fraction), LVFS (left ventricular fractional shortening), LVEDV/LVESV (left ventricular end diastolic/systolic volume), LVEDD/LVESD (left ventricular end diastolic/systolic diameter), GFP (green fluorescence protein), VEGF (vascular endothelial growth factor), COL (collage), MMP(matrix metalloproteinase), TIMP (tissue inhibitor of matrix metalloproteinase), WGA (wheat germ agglutinin), ANP (atrial natriuretic peptide), BNP (brain natriuretic peptide), MYH (myosin heavy chain), Tregs (regulatory T cells), CCL (C-C Motif Chemokine Ligand), CCR (C-C Motif Chemokine Receptor).

\section{Declarations}

\section{Ethics approval and consent to participate}

All experiments involving mice were approved by the Institutional Animal Care and Use Committee of Tongji University (Shanghai, China; approval no: TJLAC-019-131). The human umbilical cords were obtained from full-term births and implemented following the approval of Ethic Committee and the informed consent of donors in Shanghai East Hospital.

\section{Consent for publication}

Not applicable.

\section{Availability of Data and Materials}

The data that support the findings of this study are available on request from the corresponding author.

\section{Funding}

The study was supported by The National Key Research and Development Program of China (2017YFA0105600; 2017YFA0105604), the National Natural Science Foundation of China (81670458, 81500207, 81770094), The Science and Technology Commission of Shanghai Municipality (17431906600), Shanghai Engineering Research Center of Artificial Heart and Heart Failure Medicine (N0.19DZ2251000), Major Program of Development Fund for Shanghai Zhangjiang National Innovation Demonstration Zone (grant nos. ZJ2018-ZD-004) and Peak Disciplines (Type IV) of Institutions of Higher Learning in Shanghai. 


\section{Authors' contributions}

The author's contributions were as follows. $X Z$ and ZL contributed to the conception and design of the study. JL, XL, ML, XM and RZ performed the experiment. JL, XL, ML, FL, YX, QM, QZ and WH contributed to acquisition, analysis and interpretation of the data. $\mathrm{JL}$ and $\mathrm{XL}$ wrote the manuscript. $\mathrm{XZ}, \mathrm{XL}$ and $\mathrm{ZH}$ revised the MS. All authors read and approved the final manuscript.

\section{Acknowledgement}

The authors thank Dr. Ying Lu (Clinical Translational Medical Research Center, Shanghai East Hospital, Shanghai) for excellent technical assistance in flow cytometry analysis.

\section{Competing interests}

The authors have no conflicting interest associated with this manuscript.

\section{Author details}

${ }^{1}$ Research Center for Translational Medicine, Shanghai East Hospital, Tongji University School of Medicine, Shanghai 200120, P.R. China; ${ }^{2}$ Department of Burn \& Plastic Surgery, Beijing Children's Hospital, Capital Medical University, National Center for Children's Health, Beijing, 100045, P.R. China; ${ }^{3}$ Institute for Regenerative Medicine, Shanghai East Hospital, School of Life Sciences and Technology, Tongji University, Shanghai 200120, P.R. China; ${ }^{4}$ Shanghai Heart Failure Research Center, Shanghai East Hospital, Tongji University School of Medicine, Shanghai 200120, P.R. China;

${ }^{5}$ Department of Cardiovascular Surgery, Shanghai East Hospital, Tongji University School of Medicine, Shanghai 200120, P.R. China; ${ }^{6}$ Department of Heart Failure, Shanghai East Hospital, Tongji University School of Medicine, Shanghai 200120, P.R. China; ${ }^{7}$ Shanghai Institute of Stem Cell Research and Clinical Translation, Shanghai 200120, China

\section{References}

1. Moran AE, MH Forouzanfar, GA Roth, GA Mensah, M Ezzati, A Flaxman, CJ Murray and M Naghavi. (2014). The global burden of ischemic heart disease in 1990 and 2010: the Global Burden of Disease 2010 study. Circulation 129:1493-501.

2. Barron CC, MM Lalu, DJ Stewart, D Fergusson, H Yang, D Moher, P Liu, D Mazer, PJ Devereaux and L Mclntyre. (2017). Assessment of safety and efficacy of mesenchymal stromal cell therapy in preclinical models of acute myocardial infarction: a systematic review protocol. Syst Rev 6:226. 
3. Yoo KJ, RK Li, RD Weisel, DA Mickle, ZQ Jia, EJ Kim, S Tomita and TM Yau. (2000). Heart cell transplantation improves heart function in dilated cardiomyopathic hamsters. Circulation 102:lii2049.

4. Shetty P, K Cooper and C Viswanathan. (2010). Comparison of proliferative and multilineage differentiation potentials of cord matrix, cord blood, and bone marrow mesenchymal stem cells. Asian J Transfus Sci 4:14-24.

5. Santos Nascimento D, D Mosqueira, LM Sousa, M Teixeira, M Filipe, TP Resende, AF Araújo, M Valente, J Almeida, JP Martins, JM Santos, RN Bárcia, P Cruz, H Cruz and P Pinto-do-Ó. (2014). Human umbilical cord tissue-derived mesenchymal stromal cells attenuate remodeling after myocardial infarction by proangiogenic, antiapoptotic, and endogenous cell-activation mechanisms. Stem Cell Res Ther 5:5.

6. Liu C, LN Kang, F Chen, D Mu, S Shen, K Wang, JX Hu, J Xie and B Xu. (2020). Immediate Intracoronary Delivery of Human Umbilical Cord Mesenchymal Stem Cells Reduces Myocardial Injury by Regulating the Inflammatory Process Through Cell-Cell Contact with T Lymphocytes. Stem Cells Dev 29:1331-1345.

7. Lim M, W Wang, L Liang, ZB Han, Z Li, J Geng, M Zhao, H Jia, J Feng, Z Wei, B Song, J Zhang, J Li, T Liu, F Wang, T Li, J Li, Y Fang, J Gao and Z Han. (2018). Intravenous injection of allogeneic umbilical cord-derived multipotent mesenchymal stromal cells reduces the infarct area and ameliorates cardiac function in a porcine model of acute myocardial infarction. Stem Cell Res Ther 9:129.

8. Musialek P, A Mazurek, D Jarocha, L Tekieli, W Szot, M Kostkiewicz, RP Banys, M Urbanczyk, A Kadzielski, M Trystula, J Kijowski, K Zmudka, P Podolec and M Majka. (2015). Myocardial regeneration strategy using Wharton's jelly mesenchymal stem cells as an off-the-shelf 'unlimited' therapeutic agent: results from the Acute Myocardial Infarction First-in-Man Study. Postepy Kardiol Interwencyjnej 11:100-7.

9. Weirather J, UD Hofmann, N Beyersdorf, GC Ramos, B Vogel, A Frey, G Ertl, T Kerkau and S Frantz. (2014). Foxp3+ CD4+ T cells improve healing after myocardial infarction by modulating monocyte/macrophage differentiation. Circ Res 115:55-67.

10. Luger D, MJ Lipinski, PC Westman, DK Glover, J Dimastromatteo, JC Frias, MT Albelda, S Sikora, A Kharazi, G Vertelov, R Waksman and SE Epstein. (2017). Intravenously Delivered Mesenchymal Stem Cells: Systemic Anti-Inflammatory Effects Improve Left Ventricular Dysfunction in Acute Myocardial Infarction and Ischemic Cardiomyopathy. Circ Res 120:1598-1613.

11. Liu J, Q Meng, X Liang, R Zhuang, D Yuan, X Ge, H Cao, F Lin, X Gong, H Fan, B Wang, X Zhou and Z Liu. (2020). A novel small molecule compound VCP979 improves ventricular remodeling in murine models of myocardial ischemia/reperfusion injury. Int J Mol Med 45:353-364.

12. Liang $X, Y$ Ding, $F$ Lin, $Y$ Zhang, $X$ Zhou, Q Meng, X Lu, G Jiang, H Zhu, Y Chen, Q Lian, H Fan and Z Liu. (2019). Overexpression of ERBB4 rejuvenates aged mesenchymal stem cells and enhances angiogenesis via PI3K/AKT and MAPK/ERK pathways. Faseb j 33:4559-4570. 
13. Deng R, Y Liu, H He, H Zhang, C Zhao, Z Cui, Y Hong, X Li, F Lin, D Yuan, X Liang and Y Zhang. (2020). Haemin pre-treatment augments the cardiac protection of mesenchymal stem cells by inhibiting mitochondrial fission and improving survival. J Cell Mol Med 24:431-440.

14. Ong SB, S Hernández-Reséndiz, GE Crespo-Avilan, RT Mukhametshina, XY Kwek, HA Cabrera-Fuentes and DJ Hausenloy. (2018). Inflammation following acute myocardial infarction: Multiple players, dynamic roles, and novel therapeutic opportunities. Pharmacol Ther 186:73-87.

15. Squillaro T, G Peluso and U Galderisi. (2016). Clinical Trials With Mesenchymal Stem Cells: An Update. Cell Transplant 25:829-48.

16. Ulus AT, C Mungan, M Kurtoglu, FT Celikkan, M Akyol, M Sucu, M Toru, SS Gul, O Cinar and A Can. (2020). Intramyocardial Transplantation of Umbilical Cord Mesenchymal Stromal Cells in Chronic Ischemic Cardiomyopathy: A Controlled, Randomized Clinical Trial (HUC-HEART Trial). Int J Stem Cells 13:364-376.

17. Ali SR, W Ahmad, N Naeem, A Salim and I Khan. (2020). Small molecule 2'-deoxycytidine differentiates human umbilical cord-derived MSCs into cardiac progenitors in vitro and their in vivo xeno-transplantation improves cardiac function. Mol Cell Biochem 470:99-113.

18. Gyöngyösi M, J Blanco, T Marian, L Trón, O Petneházy, Z Petrasi, R Hemetsberger, J Rodriguez, G Font, IJ Pavo, I Kertész, L Balkay, N Pavo, A Posa, M Emri, L Galuska, DL Kraitchman, J Wojta, K Huber and D Glogar. (2008). Serial noninvasive in vivo positron emission tomographic tracking of percutaneously intramyocardially injected autologous porcine mesenchymal stem cells modified for transgene reporter gene expression. Circ Cardiovasc Imaging 1:94-103.

19. Hofmann M, KC Wollert, GP Meyer, A Menke, L Arseniev, B Hertenstein, A Ganser, WH Knapp and H Drexler. (2005). Monitoring of bone marrow cell homing into the infarcted human myocardium. Circulation 111:2198-202.

20. Li L, X Chen, WE Wang and C Zeng. (2016). How to Improve the Survival of Transplanted Mesenchymal Stem Cell in Ischemic Heart? Stem Cells Int 2016:9682757.

21. Yigman Z, ED Ozdemir, NN Turan, AT Ulus and A Can. (2020). Umbilical cord mesenchymal stromal cells engraft and transdifferentiate into cardiomyocyte-like cells following acute myocardial ischemia *. Acta Histochem 122:151578.

22. Liu CB, H Huang, P Sun, SZ Ma, AH Liu, J Xue, JH Fu, YQ Liang, B Liu, DY Wu, SH Lü and XZ Zhang. (2016). Human Umbilical Cord-Derived Mesenchymal Stromal Cells Improve Left Ventricular Function, Perfusion, and Remodeling in a Porcine Model of Chronic Myocardial Ischemia. Stem Cells Transl Med 5:1004-13.

23. Rai V, P Sharma, S Agrawal and DK Agrawal. (2017). Relevance of mouse models of cardiac fibrosis and hypertrophy in cardiac research. Mol Cell Biochem 424:123-145.

24. Chen YF, MA Shibu, MJ Fan, MC Chen, VP Viswanadha, YL Lin, CH Lai, KH Lin, TJ Ho, WW Kuo and CY Huang. (2016). Purple rice anthocyanin extract protects cardiac function in STZ-induced diabetes rat hearts by inhibiting cardiac hypertrophy and fibrosis. J Nutr Biochem 31:98-105. 
25. Hofmann U, N Beyersdorf, J Weirather, A Podolskaya, J Bauersachs, G Ertl, T Kerkau and S Frantz. (2012). Activation of CD4+ T lymphocytes improves wound healing and survival after experimental myocardial infarction in mice. Circulation 125:1652-63.

26. Yuan D, J Tie, Z Xu, G Liu, X Ge, Z Wang, X Zhang, S Gong, G Liu, Q Meng, F Lin, Z Liu, H Fan and X Zhou. (2019). Dynamic Profile of CD4(+) T-Cell-Associated Cytokines/Chemokines following Murine Myocardial Infarction/Reperfusion. Mediators Inflamm 2019:9483647.

27. Dayan V, G Yannarelli, F Billia, P Filomeno, XH Wang, JE Davies and A Keating. (2011). Mesenchymal stromal cells mediate a switch to alternatively activated monocytes/macrophages after acute myocardial infarction. Basic Res Cardiol 106:1299-310.

28. Tang TT, J Yuan, ZF Zhu, WC Zhang, H Xiao, N Xia, XX Yan, SF Nie, J Liu, SF Zhou, JJ Li, R Yao, MY Liao, X Tu, YH Liao and X Cheng. (2012). Regulatory T cells ameliorate cardiac remodeling after myocardial infarction. Basic Res Cardiol 107:232.

29. Bernardini G, D Ribatti, G Spinetti, L Morbidelli, M Ziche, A Santoni, MC Capogrossi and M Napolitano. (2003). Analysis of the role of chemokines in angiogenesis. J Immunol Methods 273:83101.

30. Suffee N, B Richard, H Hlawaty, O Oudar, N Charnaux and A Sutton. (2011). Angiogenic properties of the chemokine RANTES/CCL5. Biochem Soc Trans 39:1649-53.

31. Dobaczewski M, Y Xia, M Bujak, C Gonzalez-Quesada and NG Frangogiannis. (2010). CCR5 signaling suppresses inflammation and reduces adverse remodeling of the infarcted heart, mediating recruitment of regulatory T cells. Am J Pathol 176:2177-87.

32. Najar M, G Raicevic, H Fayyad-Kazan, C De Bruyn, D Bron, M Toungouz and L Lagneaux. (2013). Impact of different mesenchymal stromal cell types on T-cell activation, proliferation and migration. Int Immunopharmacol 15:693-702.

\section{Figures}

\section{Figure 1}

The safety evaluation of HucMSCs in vivo. (A) Representative images of subcutaneous tumor formation between HucMSC and A549 groups on day 60. (B) The survival curves of HucMSC and A549 groups were observed for 120 days. ${ }^{*} \mathrm{p}<0.05$ versus $A 549$ group. (C) Representative H\&E histological photos of the lung, liver, kidney and spleen on day 28. Scale bar=100 $\mu \mathrm{m}$. (D) The AST, ALT, Cr, LDH, CK, CK-MB levels in serum were measured in different groups on day 7 or 28 . ns, no significant difference versus MI-N.S group. MI, myocardiac infarct; N.S, normal saline; HucMSCs, Human umbilical cord-derived mesenchymal stem cells; SEM, Standard Error of Mean; AST, aspartate transaminase; ALT, alanine aminotransferase; $\mathrm{Cr}$, Creatine; LDH, Lactate dehydrogenase, CK, Creatine kinase; CK-MB, Creatine kinase isoenzyme. 


\section{Figure 2}

HucMSCs administration improved cardiac function of mice post MI injury. (A) The flow diagram of the experiment design. The two groups: MI-N.S and MI-HucMSC. After MI injury was established, mice were immediately injected N.S or HucMSCs by three points into the areas adjacent to the infarcted heart. (B) Representative echocardiographic images (M-mode) in two groups on day 7 following MI surgery. (C) Statistical results of cardiac function on day 7 between MI-N.S and MI-HucMSC groups ( $n=13$ per group).

(D) Representative echocardiographic images (M-mode) in two groups on day 28. (E) Statistical results of cardiac function on day 28 between MI-N.S and MI-HucMSC groups ( $n=13$ per group) following MI surgery. ${ }^{*} p<0.05$ versus MI-N.S group. MI, myocardiac infarct; N.S, normal saline; HucMSCs, Human umbilical cord-derived mesenchymal stem cells; SEM, Standard Error of Mean; ns, no significant difference versus MI-N.S; LVEF, left ventricular ejection fraction; LVFS, left ventricular fractional shortening; LVEDV/LVESV, left ventricular end diastolic/systolic volume; LVEDD/LVESD, left ventricular end diastolic/systolic diameter.

\section{Figure 3}

Retention of HucMSCs in vivo. (A) The fluorescence intensity of DiR-labeled HucMSCs were detected in multiple organs including heart, lung, liver, spleen and kidney on day 7 after MI. (B) Compared with MI-N.S group, Alu-sx was positively expressed in MI-HucMSC group by genomic PCR. Neg con, negative control; Pos con, positive control; PCR, ploymerase chain reaction. (C) Representative immunofluorescence images of GFP-HucMSC hearts on day 7. GFP, green fluorescence protein. (D) Representative immunofluorescence images of MI-HucMSC hearts stained with human mitochondrion on day 7 and 28. Green, human mitochondrion; Blue, DAPI. Scale bar=100 $\mu \mathrm{m}$. MI, myocardiac infarct; N.S, normal saline; HucMSCs, Human umbilical cord-derived mesenchymal stem cells.

\section{Figure 4}

HucMSCs treatment contributed to the angiogenesis, attenuated myocardial fibrosis and hypertrophy in hearts after MI. (A) Representative immunofluorescence images of hearts stained with CD31 between MIN.S and MI-HucMSC groups on day 7 and 28. Green, CD31; Blue, DAPI. Scale bar=100 $\mu \mathrm{m}$. (B) Statistical results of the CD31 density (per field) of MI-N.S and MI-HucMSC groups on day 7 and 28. (C) The mRNA expression of VEGF-a in infarcted hearts of two groups on day 7. (D) Representative Masson's Trichromestained histological sections of hearts between MI-N.S and MI-HucMSC groups. Scale bar $=1000 \mu \mathrm{m}$. (E) The fibrosis area of heart tissues was measured using ImageJ software. (F) The mRNA levels of COL I, COL II, MMP-2 and TIMP2 in infarcted hearts of two groups. (G) Representative immunofluorescence images of hearts stained with WGA between MI-N.S and MI-HucMSC groups. Green, WGA; Blue, DAPI. Scale bar $=50 \mu \mathrm{m} .(\mathrm{H})$ The cross-sectional areas of heart sections were estimated using ImageJ software. (I) The mRNA levels of ANP, BNP, MYH7 and MYH6 in infarcted hearts of two groups. ${ }^{*} p<0.05,{ }^{* *} p<0.01$, 
${ }^{\star * \star} p<0.001$ versus MI-N.S group. MI, myocardiac infarct; N.S, normal saline; HucMSCs, Human umbilical cord-derived mesenchymal stem cells; SEM, Standard Error of Mean. VEGF, vascular endothelial growth factor; COL, collage; MMP, matrix metalloproteinase; TIMP, tissue inhibitor of matrix metalloproteinase; WGA, wheat germ agglutinin. ANP, atrial natriuretic peptide; BNP, brain natriuretic peptide, MYH, myosin heavy chain.

\section{Figure 5}

Increase of immune cells in the infarcted heart after HucMSCs treatment on day 7. (A) Representative immunofluorescence images of hearts stained with human mitochondrion and CD3/CD4 in MI-HucMSC groups. Green, HucMSC; Red, CD3/CD4; Blue, DAPI. Scale bar=50 $\mu$ m. (B) Representative immunofluorescence images of hearts stained with CD3 and CD4 between MI-N.S and MI-HucMSC groups. Green, CD3; Red, CD4; Blue, DAPI. Scale bar=75 $\mu \mathrm{m}$. (C) Statistical results of percent of CD3+ or CD4+ T cells between MI-N.S and MI-HucMSC groups. (D) Representative immunofluorescence images of hearts stained with CD4 and FoxP3 between MI-N.S and MI-HucMSC groups. Green, CD4; Red, FoxP3; Blue, DAPI. Yellow arrow indicates CD4+FoxP3+ double positive cells. Scale bar $=100 \mu \mathrm{m}$. (E) Statistical results of cells counts of CD4+FoxP3+ Tregs between MI-N.S and MI-HucMSC groups. (F) Representative immunofluorescence images of hearts stained with CD4 and Ki67 between MI-N.S and MI-HucMSC groups. Green, CD4; Red, Ki67; Blue, DAPI. Yellow arrow indicates CD4+Ki67+ double positive cells. Scale bar=75 $\mu \mathrm{m}$. (G) Statistical results of cells count of CD4+Ki67+ T cells between MI-N.S and MI-HucMSC

groups. $(\mathrm{H})$ The flow diagram of the heart digestion and strategy of flow cytometry analysis. (I) Statistical results of cells count of immune cells between MI-N.S and MI-HucMSC groups. ${ }^{*} p<0.05$ and ${ }^{* *} p<0.01$ versus MI-N.S group. ns, no significant difference versus MI-N.S group. MI, myocardiac infarct; N.S, normal saline; HucMSCs, Human umbilical cord-derived mesenchymal stem cells; SEM, Standard Error of Mean.

\section{Figure 6}

HucMSCs promoted CD4+ T cells migration into the injured heart via high levels of CCL5 post MI. (A) Statistical results of the T cells associated cytokines and chemokines in the heart between MI-N.S and MI-HucMSC groups on day 7. (B, C) The protein and mRNA levels of CCL2 and CCL5 in heart tissues of two groups on day 7. All data were expressed as the means \pm SEM, ${ }^{*}<<0.05$ and ${ }^{* \star *} p<0.001$ versus MIN.S group. (D) In vitro, statistical results of migrated CD4+ T cells number via different treatments. $\# \# p<0.01$ versus sham group, \&\&\&p<0.001 versus MI-N.S group, ${ }^{\star} p<0.05$ versus MI-HucMSC group. MI, myocardiac infarct; N.S, normal saline; HucMSCs, Human umbilical cord-derived mesenchymal stem cells; SEM, Standard Error of Mean.

\section{Figure 7}


Schematic diagram. Intramyocardial injection of HucMSCs upregulated the CD4+FoxP3+ Tregs and contributed to the migration of CD4+ T cells into the injured heart via CCL5/CCR5 pathway. MI, myocardiac infarct; N.S, normal saline; HucMSCs, Human umbilical cord-derived mesenchymal stem cells; Tregs, regulatory T cells; CCL, C-C Motif Chemokine Ligand; CCR, C-C Motif Chemokine receptor.

\section{Supplementary Files}

This is a list of supplementary files associated with this preprint. Click to download.

- Supplementalmaterial.pdf 